\title{
Die neure Kultur des elektronischen Publizierens
}

Dr. Peter Schirmbacher

schirmbacher@cms.hu-berlin.de
Der freie ungehinderte Zugang zu wissenschaftlicher Information ist zwangsläufig nur über das Internet realistisch. Die OpenAccess-Idee ist somit untrennbar mit dem elektronischen Publizieren verbunden. Während sich in den mehr als fünfhundert Jahren print-orientierten wissenschaftlichen Publizierens feste Regeln und Verhaltensweisen der handelnden Akteure herausgebildet haben, bedarf es für das elektronische Publizieren einer Anpassung an die technischen Gegebenheiten, um die innewohnenden Vorteile ausschöpfen zu können. Der Artikel gibt Anstöße für notwendige Änderungen in der Technologie und ihrer Handhabung. (Der Beitrag ist ein stark gekürzter Auszug aus der Veröffentlichung des Autors in [1].)

\section{Elektronisches Publizieren an der Humboldt-Universität}

Das vorliegende Heft des CMS-Journals hat sich zum Ziel gesetzt, zum einen die Ideen der Open-Access-Initiative weit mehr als bisher in die Universität zu tragen und eine universitäre Diskussion anzuregen und zum anderen die im $\mathrm{Zu}$ sammenhang mit Open-Access-Publikationen stehenden technischen Parameter einer elektronischen Veröffentlichung genauer zu beschreiben. Seit I997 existiert im Computer- und Medienservice eine Arbeitsgruppe zum elektronischen Publizieren, aus der im Jahre 2002 eine gemeinsame Gruppe von Universitätsbibliothek und Computer- und Medienservice gebildet wurde. Durch diese Arbeitsgruppe »Elektronisches Publizieren« wird der hier schon mehrfach erwähnte edoc-Server technisch betreut und durch die eingeworbenen Drittmittel im Rahmen von Projekten in seinem Angebot weiterentwickelt. Die nachfolgenden Beiträge des Heftes spiegeln den Entwicklungsstand des edoc-Servers und vor allem die Möglichkeiten der Unterstützung des elektronischen Publizierens für Autoren und auch Herausgeber wider. In den Jahren der Beschäftigung mit dem elektronischen Publizieren ist an vielen Stellen deutlich geworden, dass es erhebliche Unterschiede gibt zwischen dem traditionellen papierorientierten wissenschaftlichen Publizieren und dem elektronischen Publizieren. In dem vorliegenden Beitrag sollen die Veränderungen im Publikationsprozess deutlich gemacht und damit auch ein Einblick gegeben werden, wie viel Entwicklungsarbeit $\mathrm{zu}$ leisten ist, um eine angemessene Grundlage für das elektronische Publizieren zu schaffen.

\section{Die Kultur des Publizierens}

Zweifellos gibt es die vielfältigsten Definitionen des Begriffes >Kultur< [2, 3]. Zunächst auf den Landbau und die Urbarmachung des Bodens bezogen, wird heute Kultur vielfach als die Gesamtheit des vom Menschen Geschaffenen und den dort innewohnenden Beziehungen betrachtet. Die Menschen haben sich im Verlauf der Entwicklung explizite oder implizite Regeln des Zusammenlebens und der Kommunikation geschaffen, $\mathrm{zu}$ denen zweifellos die Sprache, aber auch Werkzeuge und vor allem Verhaltensregeln gehören. O. Weggel [4] schreibt: »Kultur ist die Gesamtheit der erlernten Verhaltensweisen und der übernommenen Einstellungen, Wertesysteme und Kenntnisse (...), die von Mitgliedern einer Großgruppe geteilt und tradiert werden. Kultur ist sowohl Ausdruck als auch Bedingungsstruktur für das Verhalten der Mitglieder einer bestimmten Gesellschaft.« Akzeptiert man diese Art des Herangehens an den Kulturbegriff, so ist es sicher auch legitim, von einer Kultur der wissenschaftlichen Kommunikation bzw. des wissenschaftlichen Publizierens zu sprechen.

Unter der Kultur des Publizierens werden dabei sowohl die Gesamtheit der Verhaltensweisen des Zusammenwirkens der in den Publikationskreislauf involvierten Menschen als auch die dabei geltenden Regeln verstanden.

Sicher hilft eine solche Definition zunächst nicht weiter, den Prozess des Publizierens in seiner Vielschichtigkeit beginnend bei der Schaffung eines Manuskriptes, seiner Bearbeitung, der Veröffentlichung, der Verbreitung, der Aufbereitung oder Erschließung, der Verarbeitung durch Dritte und möglicherweise 
der Gewährleitung der Langzeitverfügbarkeit - besser zu beherrschen. Sie wird jedoch hilfreich, wenn der Prozess der Veränderungen Gegenstand der Betrachtung ist. Es sollte deutlich werden, dass bei einer Veränderung der Kultur sowohl eine Veränderung der Verhaltensweisen der an diesem Prozess beteiligten Menschen als auch eine Veränderung der bisher geltenden Regeln stattfindet.

Das wissenschaftliche Publizieren ist zweifellos die Hauptform der Darstellung wissenschaftlicher Ergebnisse. $\mathrm{Ob}$ in Monographien, Lehrbüchern, Artikeln in Zeitschriften oder Konferenzbänden, das wissenschaftliche Publizieren auf Papier hat eine lange Tradition, die auf die Erfahrungen von Jahrhunderten zurückgeht. Es ist nicht das Ziel dieses Beitrages, die Geschichte des Publizierens aufzuarbeiten, aber sicher nimmt das Jahr I452 mit der Entwicklung des Buchdrucks durch Gutenberg darin einen ganz entscheidenden Platz ein. Spätestens seit dieser Zeit hat sich die wissenschaftliche Gemeinschaft systematisch mit dem Publizieren befasst und dabei haben sich Grundregeln sowohl für die beteiligten Menschen als auch für den Umgang mit den entstehenden Werken herausgebildet.

Der Wissenschaftler als Autor betrachtet das Publizieren, zum Beispiel in einer wissenschaftlichen Zeitschrift, als eine ganz entscheidende Zielstellung seines wissenschaftlichen Arbeitens. Es ist das Produkt seiner Arbeit und stellt seinen gesellschaftlichen Beitrag in einer Wertschöpfungskette [5] dar. Die Veröffentlichung fördert die wissenschaftliche Auseinandersetzung und soll zum allgemeinen Erkenntnisgewinn beitragen. Mit der wissenschaftlichen Publikation ist häufig die gesellschaftliche Anerkennung verbunden. Auf dieses Ziel ausgerichtet, akzeptieren die Autoren eine Vielzahl von Regelungen, die mit erheblichem Mehraufwand verbunden sind. Um veröffentlichen zu können, sind in vielen Fällen Verlagsvorgaben strikt einzuhalten.

Selbstverständlich sind weitere Personengruppen in diesen Publikationskreislauf einbezogen und unterliegen den herausgebildeten Verhaltensschemata. Sie alle haben relativ klar abgrenzbare Auf- gaben, die den auf eine papiergebundene Veröffentlichung ausgerichteten Traditionen folgen. Die unterschiedlichen Personengruppen (Autoren, Gutachter, Lektoren, Herausgeber, Bibliothekare, Leser) haben in gegenseitiger Beeinflussung den Publikationsprozess geprägt und sind zwangsläufig durch ihn geprägt worden. Sie haben für die vielfältigsten Aktionen in diesem Prozess Regeln aufgestellt und diese in Abhängigkeit von den Gegebenheiten verfeinert. Es gibt sie also, »...die Gesamtheit der erlernten Verhaltensweisen und der übernommenen Einstellungen, Wertesysteme und Kenntnisse (...), die von Mitgliedern einer Großgruppe geteilt und tradiert werden ...« [4], auch für das wissenschaftliche Publizieren.

\section{Die Kultur des elektronischen Pub- lizierens oder die Veränderungen des Publikationsprozesses}

Der Erwartungswert an das elektronische Publizieren ist sehr vielschichtig. Eine Vielzahl an Vorteilen kann sich auftun, wenn es gelingt, die modernen Methoden der Informationstechnologie adäquat einzusetzen. Notwendige Bedingung dafür ist eine Veränderung in den Handlungsweisen der beteiligten Personen. Ähnlich wie beim bisherigen Verfahren müssen sich die Autoren, die Verlage, die Bibliotheken und die Nutzer mit den Veränderungen auseinander setzen oder besser noch, sie bewusst gestalten. Das ist sicher ein Prozess, den es herauszubilden gilt. Setzt man den Beginn des elektronischen Publizierens mit der Verfügbarkeit des World Wide Web gleich, so handelt es sich um einen historisch extrem kurzen Zeitraum von I2 bis I5 Jahren. Verständlicherweise fehlen viele Regeln, Standards und fest gefügte Verhaltensweisen. Das bisherige Publizieren kann auf eine viele hundert Jahre umfassende Tradition zurückgreifen. So ist es für den Autor selbstverständlich, dass er sein Manuskript in einer den Vorgaben des Verlages entsprechenden Form abliefert, damit es dort möglichst mit geringem Aufwand weiterbearbeitet werden kann. Es gehört zum guten Ton, dass der
Verlag für den Druck die Erkenntnisse über möglichst lange haltbares Papier berücksichtigt, genauso wie es heftig diskutierte Regeln für das Erschließen einer wissenschaftlichen Arbeit in der Bibliothek gibt. Das alles sind Beispiele für die bestehende Kultur des Publizierens.

Was muss sich also ändern und wer muss sich wie ändern beim elektronischen Publizieren? Eine Dekade Entwicklungszeit ist sicher sehr wenig, sodass die folgenden Ausführungen nur eine Tendenz darstellen können. Veränderungen wird es in den folgenden Jahren in den Details sicher noch mehrfach geben. Es sei darauf hingewiesen, dass sich der vorliegende Artikel mit dem textorientierten Publizieren beschäftigt. Die multimediale Darstellung wissenschaftlicher Inhalte steckt noch in den Kinderschuhen und bedarf einer Vielzahl weiterer Untersuchungen, um zu Standards, Regeln und Verhaltensweisen zu gelangen.

Dem Autor sollte bewusst sein, dass er durch seine Verhaltensweise ganz entscheidend die Vorteile des elektronischen Publizierens unterstützen kann. Vergleichbar den bisherigen Verlagsvorgaben für Manuskripte, sollte er sich frühzeitig mit der Institution, die sein Werk für die Veröffentlichung vorbereitet, in Verbindung setzen. Das kann in gleicher Weise wie bisher ein Verlag sein, aber auch ein Publikationsservice der wissenschaftlichen Einrichtung, der er angehört. Von besonderer Wichtigkeit ist, dass der Autor ein Dateiformat benutzt, dass sich für eine Langzeitarchivierung eignet. Benutzt er ein proprietäres Format, so ist die Wahrscheinlichkeit gering, dass sein Werk in zwanzig Jahren noch lesbar sein wird. Die Arbeitsgruppe Elektronisches Publizieren der Deutschen Initiative für Netzwerkinformation hat in zwei Abhandlungen entsprechende Empfehlungen formuliert [6,7].

Neben der Vermeidung eines proprietären Dateiformats sollte der Autor durch die Widerspiegelung der Art des Schreibens dazu beitragen, dass die entstehenden Ergebnisse einem qualitativ besseren Retrieval zugeführt werden können.

Was ist damit gemeint?

Der Autor eines Zeitschriftenartikels entwickelt seine Vorstellungen über die 
Ziele des Artikels in der Mehrzahl der Fälle, in dem er sich eine Gliederung im Sinne einer inneren Struktur überlegt. Danach füllt er diese Punkte systematisch mit Inhalten und stellt möglicherweise bei der Endredaktion noch die eine oder andere Passage um. In den Gedanken des Autors hat der Artikel eine Struktur, meist geordnet nach Abschnitten, Kapiteln, Unterkapiteln u. ä. Diese Struktur ist, wenn sie auf Papier oder den Bildschirm gebracht wird, für die Menschen auch nachvollziehbar. Für Suchmaschinen stellt sich das Ergebnis der Arbeit jedoch als sequentieller Text dar, der sich maschinell nicht erschließen lässt. Man spricht dann von einem so genannten unstrukturierten Volltext. Würde der Autor jedoch während des Schreibens die Struktur des Artikels darstellen, so gäbe es später die Chance einer maschinellen Analyse. Die Struktur des Artikels in der heutigen Zeit darzustellen, bereitet mit Hilfe der modernen Textverarbeitungssysteme keine besonderen Schwierigkeiten. In jedem dieser Systeme gibt es die Möglichkeit, z. B. die Überschrift eines Kapitels als ein Strukturmerkmal hervorzuheben und in gleicher Weise auch die Überschrift eines Unterkapitels, damit sie sich von der Hauptüberschrift unterscheidet. Ich kann ein Vorwort als ein solches kennzeichnen, genauso wie eine Bild- oder Tabellenunterschrift. In ähnlicher Weise lassen sich Zitate oder Literaturverweise vom übrigen Text differenzieren. Diese besonderen Auszeichnungen von Strukturmerkmalen lassen sich zusammenfassen. Die Darstellung dieser Struktur nennt man dann eine Dokument Typ Definition (DTD - Document Type Definition). Strukturbeschreibungen lassen sich in den Auszeichnungssprachen, wie z. B. SGML (Standardized General Markup Language) oder XML (Extensible Markup Language) auch wiedergeben und können somit zu suchbaren Elementen moderner Maschinen werden. Theoretisch könnte es so sein, dass jeder Text seine eigene, sich von anderen unterscheidende Struktur hat. Die ist jedoch nicht die Praxis. So besitzen die Artikel einer Zeitschrift eine ähnliche Struktur, genauso wie die Dissertationen einer Fachrichtung (siehe hierzu auch Diss DTD [8] auf dem edoc-Server der Humboldt-Universität zu Berlin). Die Tiefe der Wiedergabe der Struktur eines wissenschaftlichen Textes kann sicher variieren, deutlich sollte jedoch werden, dass durch die Suchbarkeit von Strukturelementen eine größere Treffergenauigkeit erreicht werden kann und somit das Retrieval eine Qualitätssteigerung erfährt.

Ähnlich wie bei der traditionellen Art zu publizieren, sollte sich also der Autor mit dem Herausgeber verständigen, unabhängig davon, ob es sich um einen Verlag oder einen Publikationsservice einer wissenschaftlichen Einrichtung handelt. Vielfach existieren dort DTD's oder entsprechende Formatvorlagen (Microsoft Word) bzw. Templates (Open Office), die dann als Vorlage für die eigene Arbeit dienen können.

Diese beiden Beispiele verdeutlichen, dass der Autor durch die Veränderung seiner bisherigen Gewohnheiten beim Erstellen der wissenschaftlichen Arbeit ganz entscheidend zur Ausnutzung der Vorteile des elektronischen Publizierens beitragen kann.

Die vielfach sehr heftig geführte Diskussion zur heutigen Rolle der Verlage im Publikationsprozess soll an dieser Stelle nicht aufgenommen werden, da es in diesem Beitrag in erster Linie um die Veränderung der Verhaltensweisen derjenigen Institution gehen soll, die für die Veröffentlichung der wissenschaftlichen Arbeit verantwortlich zeichnet. Verantwortlich zeichnen bedeutet im Zusammenhang mit dem Publizieren zumindest folgende Punkte:

- Veröffentlichung und Verbreitung,

- Wahrung der Authentizität,

- Wahrung der Integrität,

- Bereitstellung für die Langzeitarchivierung.

Die bisherige Kultur des Publizierens hatte für diese Punkte fest gefügte Regelungen, die durch den Erfahrungsschatz im Umgang mit Papier seit über tausend Jahren geprägt sind. Es gibt Vertriebsstrukturen, Marketingstrategien, einen Fachbuchhandel und natürlich wissenschaftliche Bibliotheken. Vergleichbares gibt es für elektronische Medien nicht. Während eine Veröffentlichung im Internet zeitnah erfolgen kann, da der Auf- wand sich in Grenzen hält und nahezu automatisch auch die weltweite Verbreitung einschließt und damit den entscheidenden Vorteil des elektronischen Publizierens voll zum Tragen bringt, gibt es für die anderen drei Aufgabenspektren bisher keine etablierten Lösungen. Das ist, um mich zu wiederholen, bei der historisch kurzen Zeit, seit man vom elektronischen Publizieren sprechen kann, nicht verwunderlich, wichtig ist jedoch, dass man sich dieser Probleme bewusst ist, zielgerichtet an ihrer Lösung arbeitet und damit veränderte Verhaltensweisen etabliert.

Es klingt so selbstverständlich, ist es aber in der Realität sehr häufig nicht. Derjenige, der etwas ins Netz stellt, sollte sich dessen bewusst sein, dass damit eine weltweite Veröffentlichung und ein weltweiter Zugriff (wenn nicht Einschränkungen vorgesehen sind) verbunden sind. Bedauerlicherweise existieren viele Server von wissenschaftlichen Einrichtungen, einzelnen Forschergruppen oder einzelnen Wissenschaftlern, bei denen man diesen Eindruck leider nicht hat. Zur Kultur des elektronischen Publizierens gehört, dass man sich als Betreiber eines Publikationsservers bewusst ist, dass man einen weltweiten Service anbietet, der sich in das Gesamtgefüge der wissenschaftlichen Veröffentlichungen einbettet. Damit verbunden ist die Bereitschaft der Verantwortungsübernahme für die Qualität des Services. Hier ist nicht in erster Linie die inhaltliche Qualität der wissenschaftlichen Veröffentlichung gemeint, sondern die Qualität und Stabilität des Services. Der Betreiber des Servers ist z. B. verantwortlich, dass man ein einmal auf seinem Server gefundenes wissenschaftliches Werk immer wieder an derselben Stelle, unverändert und tatsächlich von dem Autor verfasst, der angegeben wurde, findet.

Im schon erwähnten DINI-Zertifikat [9] wird deshalb eine Reihe von Anforderungen an den Betreiber dieses Publikationsservices gestellt. So wird eine Server-Policy erwartet, in der Basisregeln für den Betrieb dargestellt werden. So sollten sowohl der Autor als auch der Leser aus der Policy ableiten können, welche Mechanismen der Sicherung des Servers ge- 
gen Manipulation oder Datenverlust vorgesehen sind und welche Maßstäbe bei der Wahrung der Integrität der einzelnen Dokumente gelten. Als ein Beispiel für eine Mindestanforderung an einen solchen Publikationsservice kann die Vergabe von Persistent Identifiers für jedes Dokument gelten, d.h. jedes auf dem Serve angebotene Dokument bekommt eine eindeutige Adresse, die sichert, dass das Dokument unter dieser Adresse immer erreichbar sein wird.

Während des klassischen Publikationsprozesses setzt nach der Veröffentlichung und Verbreitung die Phase der Erwerbung, Erschließung und Aufbewahrung ein, also charakteristische Aufgaben für die wissenschaftliche Bibliothek. Die Rollen sind beim elektronischen Publizieren im Wesentlichen nicht anders verteilt, nur die Grenzen zwischen den Aufgaben der Beteiligten sind noch nicht klar abgesteckt. So entwickeln zum einen wissenschaftliche Bibliotheken einen Publikationsservice als zusätzliches Angebot für die Mitglieder ihrer Einrichtung, z. B. einer Universität, und zum anderen gibt es vermehrt Verlage, die von sich behaupten, dass sie auch die Erschließung und den Nachweis der bei ihnen erschienenen wissenschaftlichen Arbeiten übernehmen, also im eingeschränkten Sinne Aufgaben einer bisherigen Bibliothek. Für den Gegenstand dieses Beitrages ist es vordergründig nicht von ausschlaggebender Bedeutung, wer die sich verändernden Aufgaben beim elektronischen Publizieren wahrnimmt, wichtig ist, dass sie bewusst aufgenommen und gestaltet werden.

Im Sinne einer neuen Kultur des elektronischen Publizierens geht es um standardisierte Metadaten, um die Austauschbarkeit dieser Daten, um die Integration des elektronisch verfügbaren Bestandes in den OPAC, um die Sicherung der Langzeitverfügbarkeit und die Wahrung der Authentizität und Integrität der Dokumente.

Die Lesenden haben diese Veränderungen des Publikationsgeschehens am schnellsten aufgegriffen und teilweise ihre Verhaltensweisen bereits geändert. So besagen Untersuchungen [10], dass heute auf elektronisch verfügbare Dokumente bis zu acht Mal häufiger zugegrif- fen wird als auf ausschließlich auf Papier erscheinende Dokumentationen. Nun sind solche Zahlen sicher etwas vorsichtig zu betrachten und variieren sehr stark in Abhängigkeit vom Fachgebiet. Von der Tendenz wird jedoch deutlich, dass der Lesende die sich ihm bietenden Möglichkeiten immer mehr nutzt und somit als forderndes Glied im Publikationsprozess zu verstehen ist. Natürlich muss aber auch er sich mit den neuen Möglichkeiten und den noch vorhandenen Grenzen auseinander setzen. Hier ist insbesondere seine Einflussnahme auf die Anbieter der Dokumente gefragt und somit sein Wirken auf die Gesamtheit des Gestaltungsprozesses. Nicht zuletzt ist er derjenige, der die gegenwärtige Diskussion über den ungehinderten Zugang zu Informationen (Open-Access-Initiative) ins Leben gerufen hat und am heftigsten dafür eintritt.

\section{Zusammenfassung und Ausblick}

Die Anwendung der modernen Methoden der Informations- und Kommunikationstechnologie führt zu einer der größten Umwälzungen innerhalb der bisherigen wissenschaftlichen Kommunikation. Das elektronische Publizieren als eine Form der wissenschaftlichen Kommunikation steht dabei ganz am Anfang seiner Entwicklung. Es gilt diesen Prozess der Veränderung zu gestalten, um die augenscheinlichen Vorteile dieser neuen Technologie auch voll zur Geltung kommen zu lassen. Wichtig ist es, dabei nicht nur ein »elektronisches Abbild « des bisherigen Publikationsprozesses zu erzeugen, sondern durch die Ausnutzung der Vorzüge der elektronischen Kommunikation den gesamten Prozess zu verändern und sich dabei auch von teilweise überholten Verhaltensweisen zu trennen. Der vorliegende Artikel ist im gewissen Sinne nur eine Momentaufnahme. Es wird die Forderung nach einer neuen Kultur des elektronischen Publizierens erhoben und dabei auf bestimmte Verhaltensweisen, einzuhaltende Mindeststandards und sich aufzeigende Entwicklungsrichtungen orientiert. Zur Vereinfachung der Darstellung wurde ausschließlich die vorrangig textorientierte wissenschaftliche
Arbeit unterstellt. Betrachtet man jedoch die gegenwärtigen Entwicklungen, so wird deutlich, dass die wissenschaftliche Kommunikation bei diesem Stadium nicht Halt machen wird, sondern der Umgang mit multimedialen Werken unser künftiges Arbeiten bestimmen wird. Die Forderung nach einer Veränderung der Verhaltensweisen aller am Publikationsprozess Beteiligten und ihrem bewussten Umgang bei der Erstellung, Erschließung und Aufbewahrung dieser »neuen« Medien wird sich verschärfen, weil nur so das wissenschaftliche Gedankengut für die Nachwelt aufbewahrt werden kann.

\section{Literatur}

[1] Die innovative Bibliothek. K.G. Saur Verlag, München 2005, S. IO7 ff.

[2] Enzyklopädie Philosophie und Wissenschaftstheorie. Hg. Jürgen Mittelstraß, Bde. I-2, Mannheim-Wien-Zürich, I980, Bde. 3-4, Stuttgart-Weimar, I995-I996.

[3] Halder, A., Müller, M.: Philosophisches Wörterbuch. Erw. Neuausgabe, Freiburg-Basel-Wien, I993.

[4] Wegged, O. : Die Asiaten. München, I989, S. 22.

[5] Roosendaal, H., Geurts, P., van DER VeT, P.:Eine neue Wertschöpfungskette für den Markt der wissenschaftlichen Information? Bibliothek 26, 2002, Nr.2, S. I49-I53.

[6] http://www.dini.de/documents/ OAI-Empfehlungen-Okt2003-de.pdf

[7] http://www.dini.de/documents/ Zertifikat.pdf

[8] http://edoc.hu-berlin.de/diml/

[9] http://www.dini.de/documents/ Zertifikat.pdf

[10] Harned, S., Brody, T., Vallieres, F., Carr, L., HitchCock, S., Gingras, Y., Oppenheim, C., Stamerjohanns, H., Hilf, E.: The green and the gold roads to Open Acces. http://www.nature.com/nature/ focus/accessdebate/2r.html 